Supporting Information

\title{
Nanodot-to-Rod Transition and Particle Attachment in Self-Organized Polycrystalline Aggregates
}

Pamela Knoll and Oliver Steinbock*

*Department of Chemistry and Biochemistry, Florida State University, Tallahassee, FL 32306-4390, United States

\section{Experimental}

Main Experiment Stock solution of barium chloride and/or strontium chloride (0.1 $\mathrm{M}$ ) along which sodium metasilicate $\left(8.85 \mathrm{mM}\right.$ ) were prepared and purged using nitrogen gas to remove dissolved $\mathrm{CO}_{2}$. The silicate solution was prepared prior to every experiment because aging of this solution could affect biomorph growth. The initial $\mathrm{pH}$ value of the crystallization solution was set to 10.7 by addition of $0.05 \mathrm{M} \mathrm{HCl}$. Each crystallization experiment was carried out in plastic Petri dishes ( $35 \mathrm{~mm}$ diameter) filled with $5 \mathrm{~mL}$ of aqueous barium chloride $(5.0 \mathrm{mM})$, sodium silicate solution $(8.4 \mathrm{mM})$, and sodium carbonate $(0.01 \mathrm{mM})$. Notice that in these experiments, the added carbonate is the initial source for metal carbonate formation but in addition $\mathrm{CO}_{2}$ enters the basic solution from the air. The Petri dishes are covered during the experiment with their lids but not further sealed. Moreover, one small plastic plate (about $1 \mathrm{~cm}^{2}$ ) is placed into each Petri dish for easy retrieval of biomorphs on substrates that can be readily used for further characterization. All experiments are performed at room temperature.

Quenching Experiments For our experiments, it is important that the crystallization front is rapidly quenched to gain insights into the structure of the active growth edge and to also allow its comparison to stalled fronts. Quenching is accomplished by rapid removal of the sample plates and immediate submersion into a dilute $\mathrm{NaOH}$ solution $(\mathrm{pH} 9)$. This $\mathrm{pH}$ was selected to ensure that both metal carbonate and silica do not dissolve. After about $10 \mathrm{~s}$, samples are then extracted again, placed into water for $30 \mathrm{~s}$, and finally dried at ambient conditions.

Solution Transfer Experiments To study specific aspects of the dot-to-rod transition, we performed some experiments in which the plastic sample carrier (the small plexiglass plate) was rapidly transferred from a barium to a strontium solution or vice versa. The two solutions had identical initial concentrations and were prepared at the same time. Transfer took less than $5 \mathrm{~s}$ and was carried out 4-5 $\mathrm{h}$ after preparation of the crystallization solution. To avoid a sudden influx of $\mathrm{CO}_{2}$, we performed this step under Ar gas. Earlier attempts without Ar showed a more pronounced disturbance of the sheet as expected from previous studies. At the end of all of these experiments, the samples were quenched by rapid transfer to a dilute $\mathrm{NaOH}$ solution (see above). The quenching was typically transformed $1 \mathrm{~h}$ after the transplantation. 
Similar experiments were also carried out on inactive biomorph sheets and the exposure time to the second solution was varied systematically between 0 and $2 \mathrm{~h}$. Again the growth was quenched but after completion of the specified exposure times.

Optical Microscopy During growth, samples were monitored using an inverted optical microscope (Leica DM IRB) at a magnification of 10x or 40x. The microscope was connected to a Nikon D3300 camera. For experiments utilizing polarized light, we equipped the microscope with two polarizing filters (Glare-Stop, Visual Pursuit, Inc., Chicago). This technique was used for both actively growing and dried samples. For the latter, we also added mineral oil (Acros Organics, density $0.877 \mathrm{~g} / \mathrm{cm}^{3}$ ) onto the sample to reduce the difference in refractive index between the sample and the surrounding medium. The respective refractive index values are for the birefringent witherite $n_{\alpha}=1.529, n_{\beta}=1.676$, and $n_{\psi}=1.677$ and for the oil $n=$ $1.46 .^{1}$

Scanning Electron Microscopy (SEM) and EDX Spectroscopy Dried samples were typically sputter-coated with a $4 \mathrm{~nm}$ layer of iridium or in a few cases with carbon. The coated samples were imaged using a FEI Nova 400 field emission scanning electron microscope (SEM) with an accelerating voltage between 5-7.5 $\mathrm{kV}$. All compositional analyses were performed by energy dispersive X-ray (EDX) spectroscopy using an Oxford INCA X-sight detector.

Transmission electron microscopy (TEM) and Selected Area Electron Diffraction (SAED) Biomorphs were grown directly on carbon-coated copper TEM grids (200 mesh, Electron Microscopy Sciences, CF200-CU) that after mixing of the reactants were placed into the solution. We monitored the progress of the biomorph growth using the inverted optical microscope (see above) and quenched the growth once a biomorph sheet had formed on the grid and grown to a sufficient size. Typically, we observed between zero and three biomorph sheets per grid. Quenching employed the procedure outlined above. The rinsed and dried samples were then investigated using a state-of-the-art JEM-ARM200cF Transmission Electron Microscope operated at $120 \mathrm{kV}$. At this high acceleration voltage, beam effects were detected for some samples and manifested themselves in fast melting-like dynamics.

\section{Movie}

Movie S1. Video obtained using optical bright-field microscopy showing an actively growing biomorph sheet. The movie demonstrates the local stalling of the crystallization front.

\section{References}

1) Lide, D. R. CRC Handbook of Chemistry and Physics $76^{\text {th }}$ Edition (CRC Press, Taylor\&Francis, Boca Raton, FL, 2007), p. 4-138. 

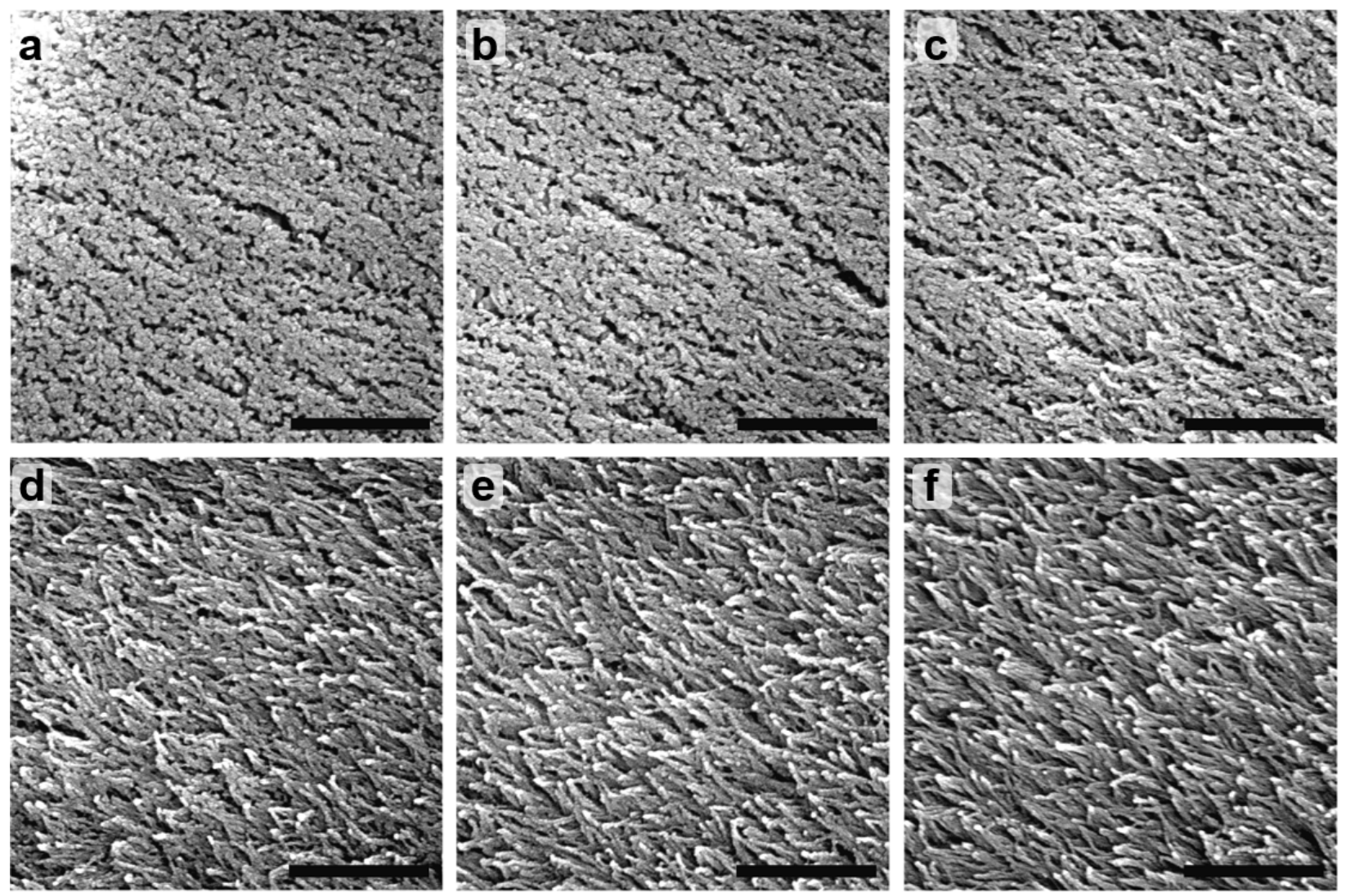

Figure S1. Series of SEM images of a quenched biomorph sheet sorted by increasing distance from the active sheet edge (closest distance in a)). All scale bars are $500 \mathrm{~nm}$. This figure provides another example of the dot-to-rod transition (see also Figure 1 ) and shows the image data used for the analysis in Figure 3a. 


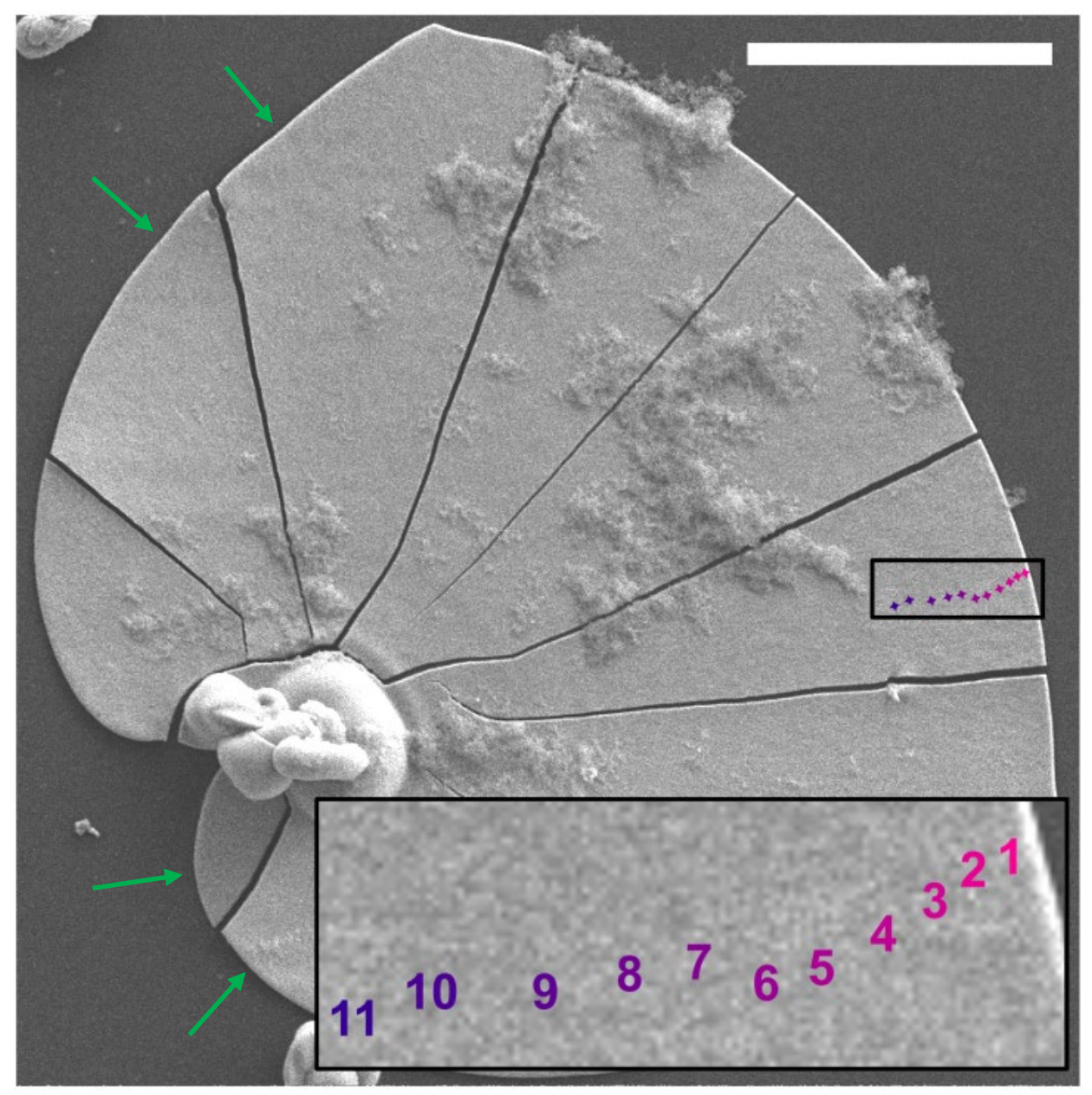

Figure S2. SEM image of a biomorph sheet quenched during growth. The marked areas were analyzed using EDX spectroscopy to determine the Ba/Si ratio with increasing distance $d$ from the actively growing front. Notice that the two spiral-shaped edges in the left portion of the image (green arrows) correspond to stalled fronts. The radial cracks occurred during the drying of the sample. Scale bar is $50 \mu \mathrm{m}$.

Table S1. Barium-to-silicon ratio from EDX measurements for the areas in Figure S2.

\begin{tabular}{|c|c|c|}
\hline$\#$ & Edge distance $\boldsymbol{d}(\boldsymbol{\mu m})$ & $\mathbf{B a} / \mathbf{S i}$ \\
\hline 1 & 0.83 & 4.2 \\
\hline 2 & 2.47 & 3.9 \\
\hline 3 & 4.08 & 3.8 \\
\hline 4 & 6.08 & 3.7 \\
\hline 5 & 8.36 & 3.9 \\
\hline 6 & 10.44 & 3.7 \\
\hline 7 & 12.67 & 3.7 \\
\hline 8 & 14.98 & 3.5 \\
\hline 9 & 17.97 & 3.7 \\
\hline 10 & 21.83 & 3.4 \\
\hline 11 & 24.61 & 3.5 \\
\hline
\end{tabular}



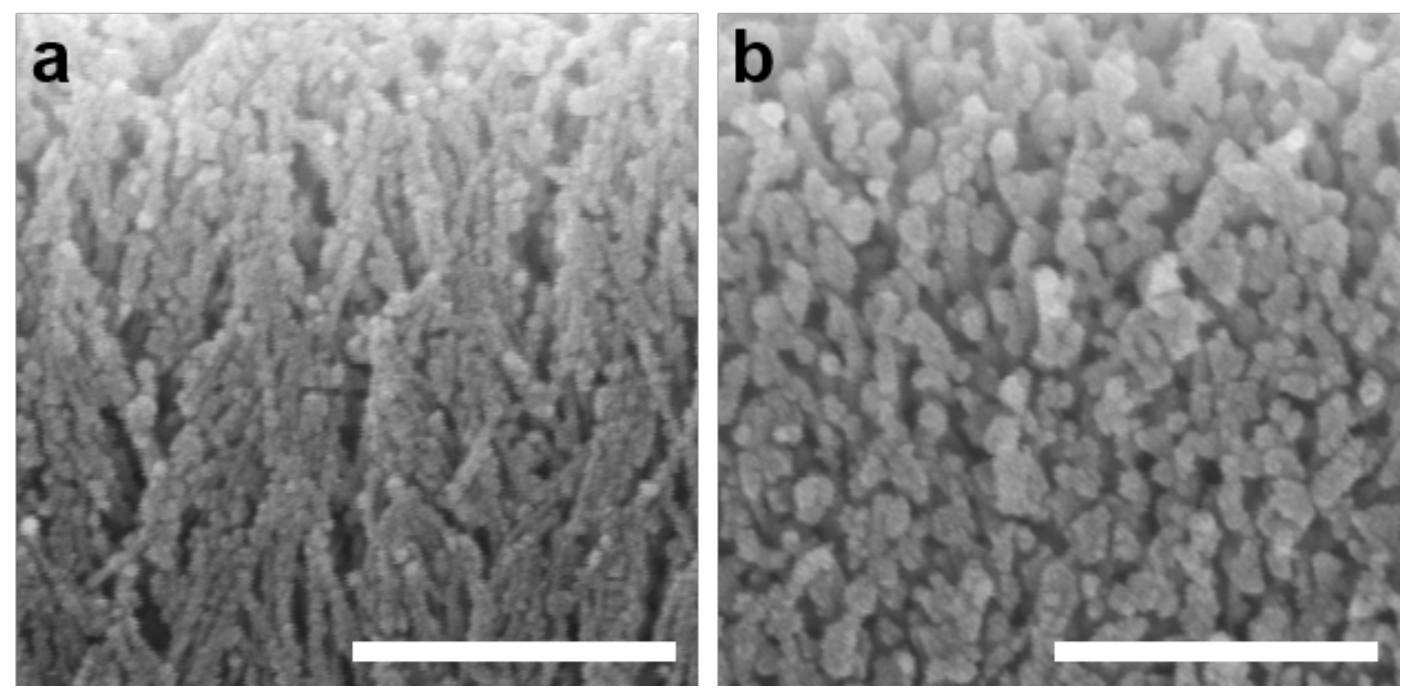

Figure S3. Scanning electron micrographs comparing biomorph sheets that formed at different times: a) early sheet edge region (quenched $4 \mathrm{~h}$ after start of reaction), b) late sheet edge region (quenched $6.5 \mathrm{~h}$ after start of reaction). The corresponding time difference $(2.5 \mathrm{~h})$ is larger than the time elapsed between the crystallization of the regions shown in Figures 1b,c of the main paper (about 50 min). Nonetheless, both SI images show nanodots rather than well-formed nanorods. The differences are small and primarily size variations (early nanodots are smaller than late ones). We hence rule out that the nanodot-tonanorod transition occurred merely due to changes in the solution rather than changes of the crystallized material. Scale bars: $300 \mathrm{~nm}$. The sheet edges are located along the upper image border just outside the field of view. Both sheet edges are quenched active fronts. 


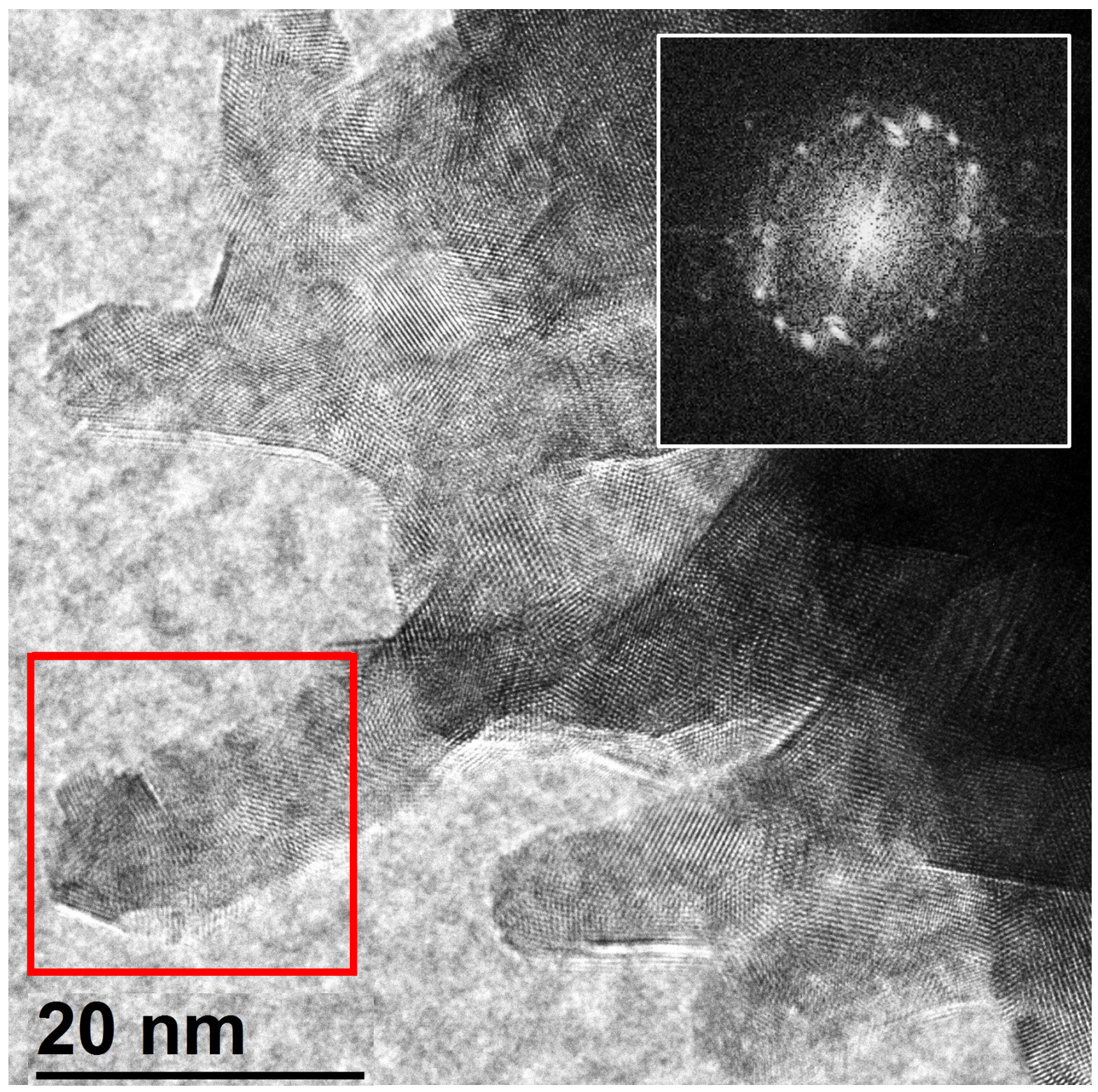

Figure S4. Second example of a transmission electron micrograph showing the nanoscale morphology along a quenched, active sheet edge. The region consists of aggregated particles with different lattice plane orientations. Nanorod-like feature are either absent or very weakly developed. The inset shows the selected area diffraction pattern of the particles in the red box. 


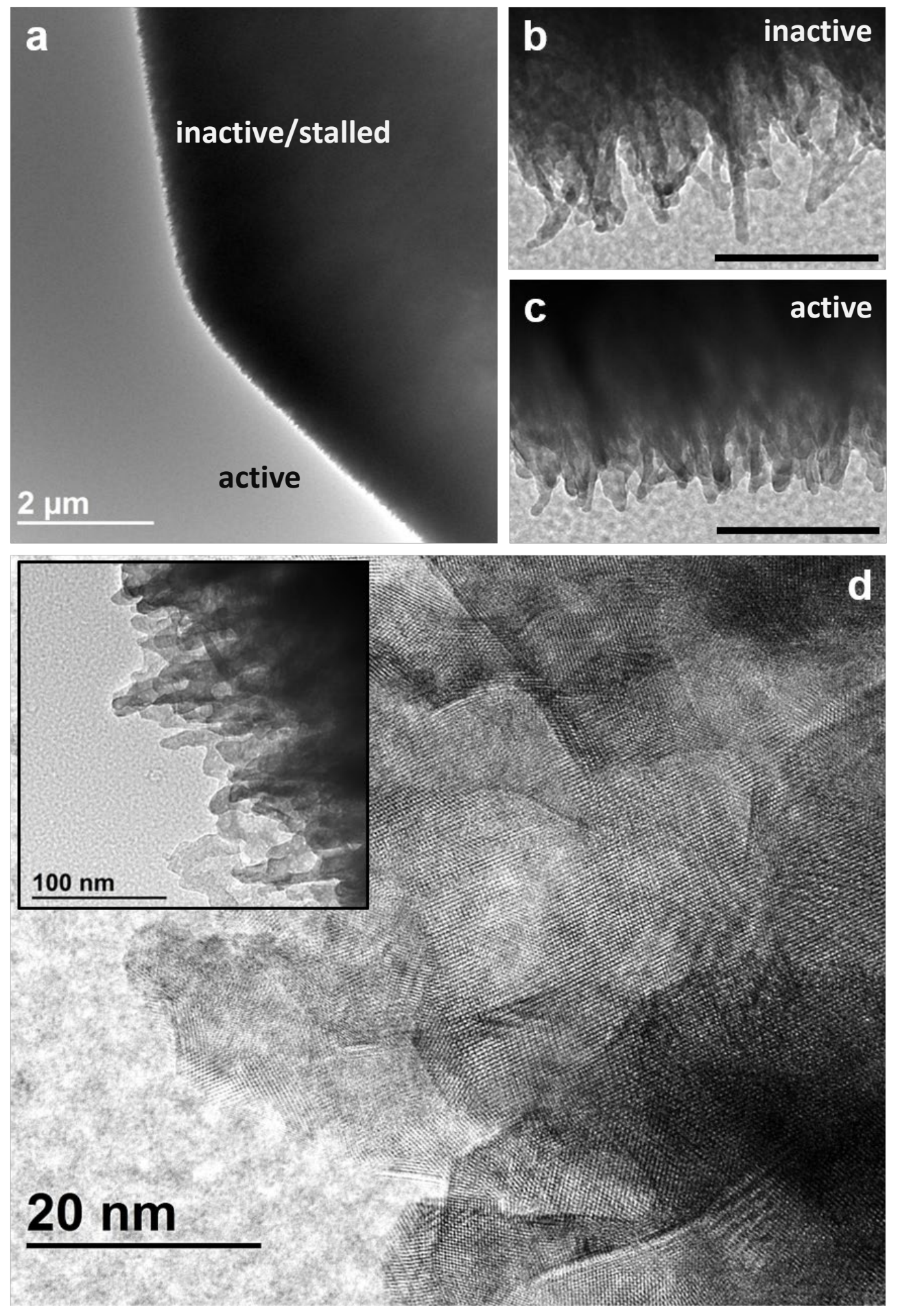

Figure S5. TEM images along a quenched sheet edge. a) Overview of the analyzed area at low magnification. The corner demarks the point at which inactive and active fronts meet. b,c) Magnified views of the inactive and active regions, respectively. Scale bars $100 \mathrm{~nm}$. d) High-resolution TEM image of the active front of a different sheet (larger view in inset). 


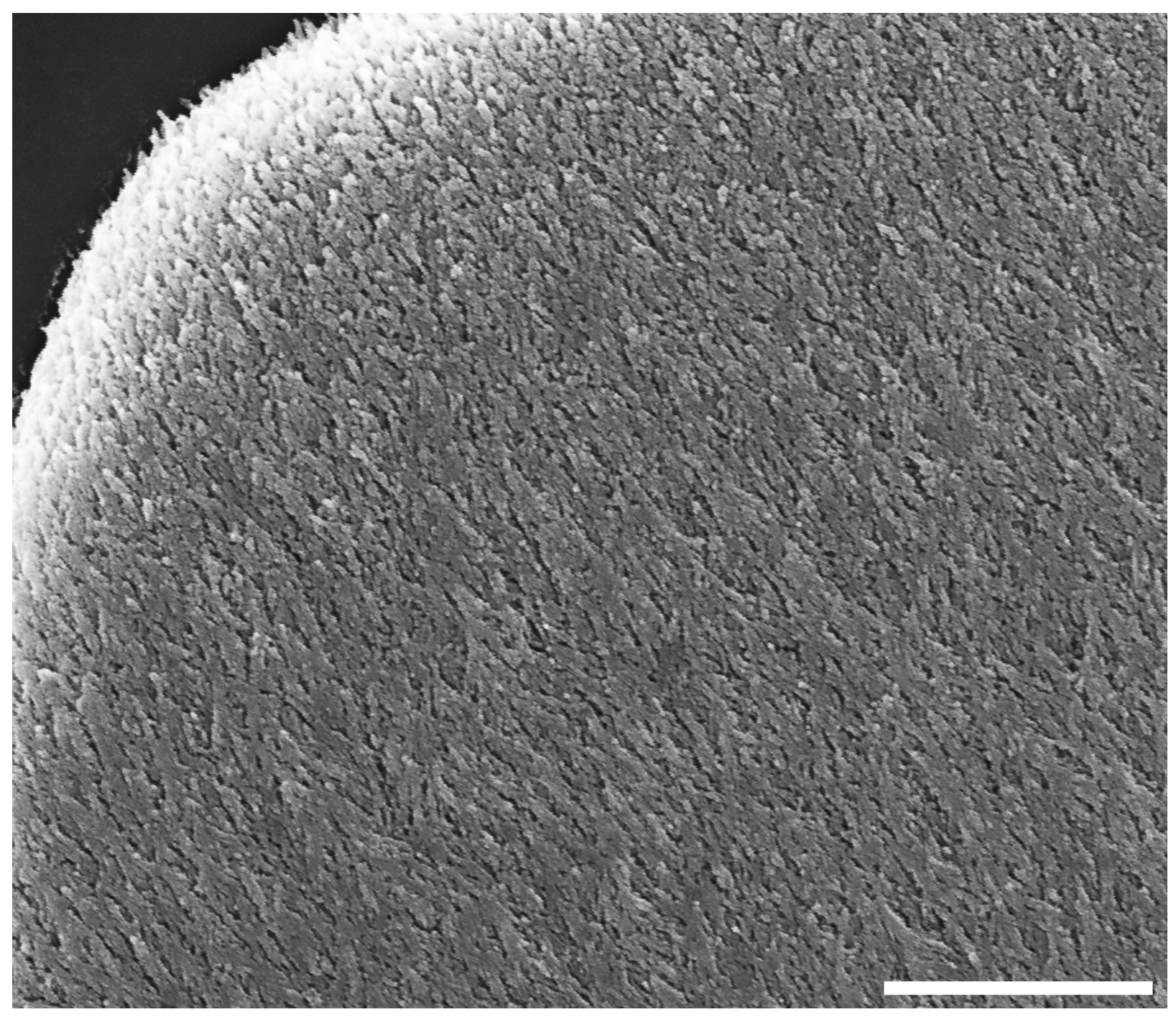

Figure S6. Larger view of Figure 3b (main paper) showing the corner of a growing sheet. Scale bar is $1 \mu \mathrm{m}$. 

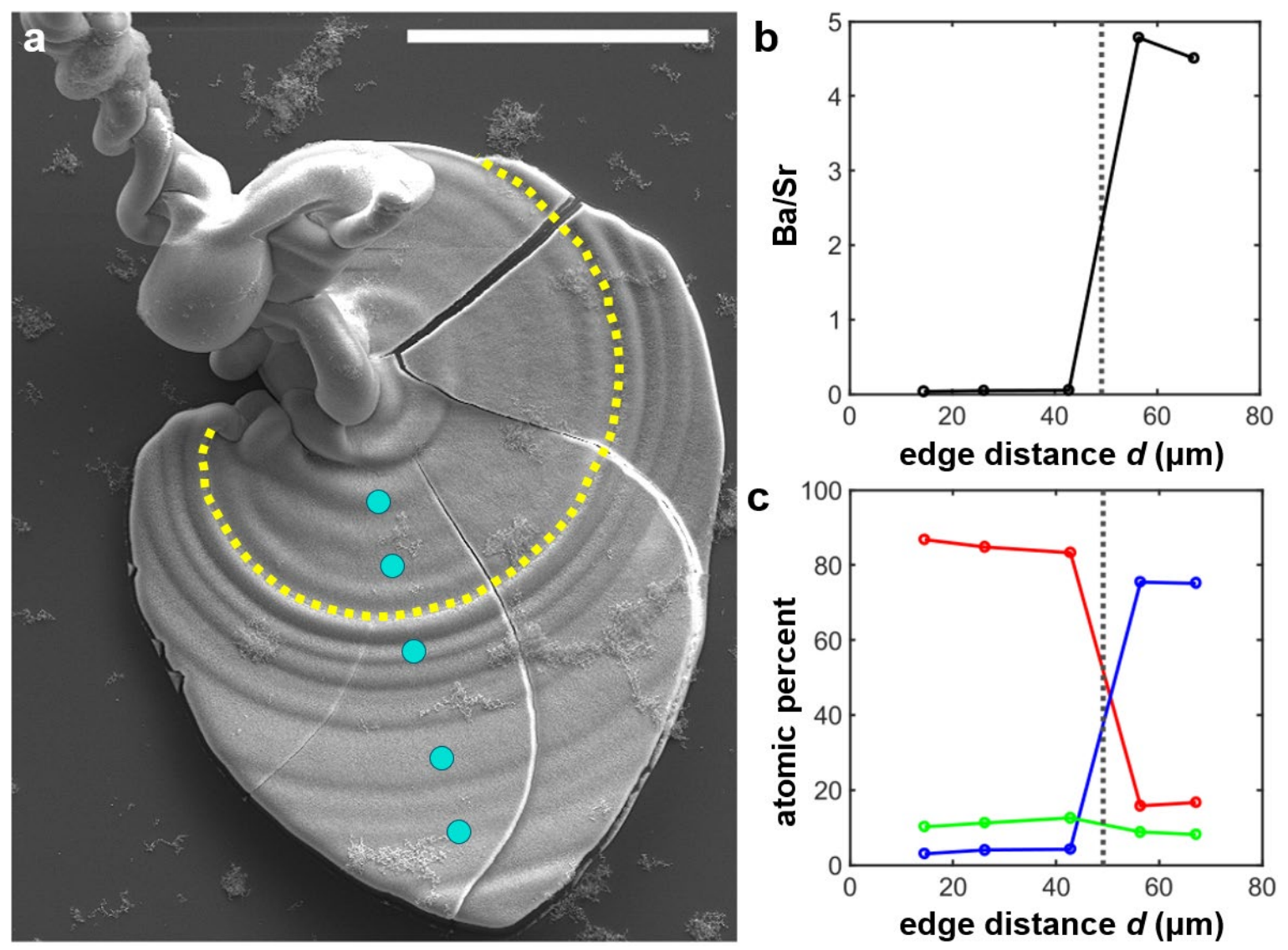

Figure S7. Compositional analysis of the Ba/Sr ratio for a biomorph sheet that was first grown in a Basolution and then rapidly transferred to a comparable Sr-solution. a) SEM image of the analyzed biomorph sheet with the dotted, yellow line indicating the transition zone and cyan circles marking the centers of the analyzed areas. Scale bar is $40 \mu \mathrm{m}$. b,c) Compositional information obtained by EDX spectroscopy as a function of the distance from the quenched, active edge. The gray dashed line marks the lip, i.e. the abrupt transition from barium to strontium. Blue, red, and green colors in c) correspond to barium, strontium, and silica, respectively. 

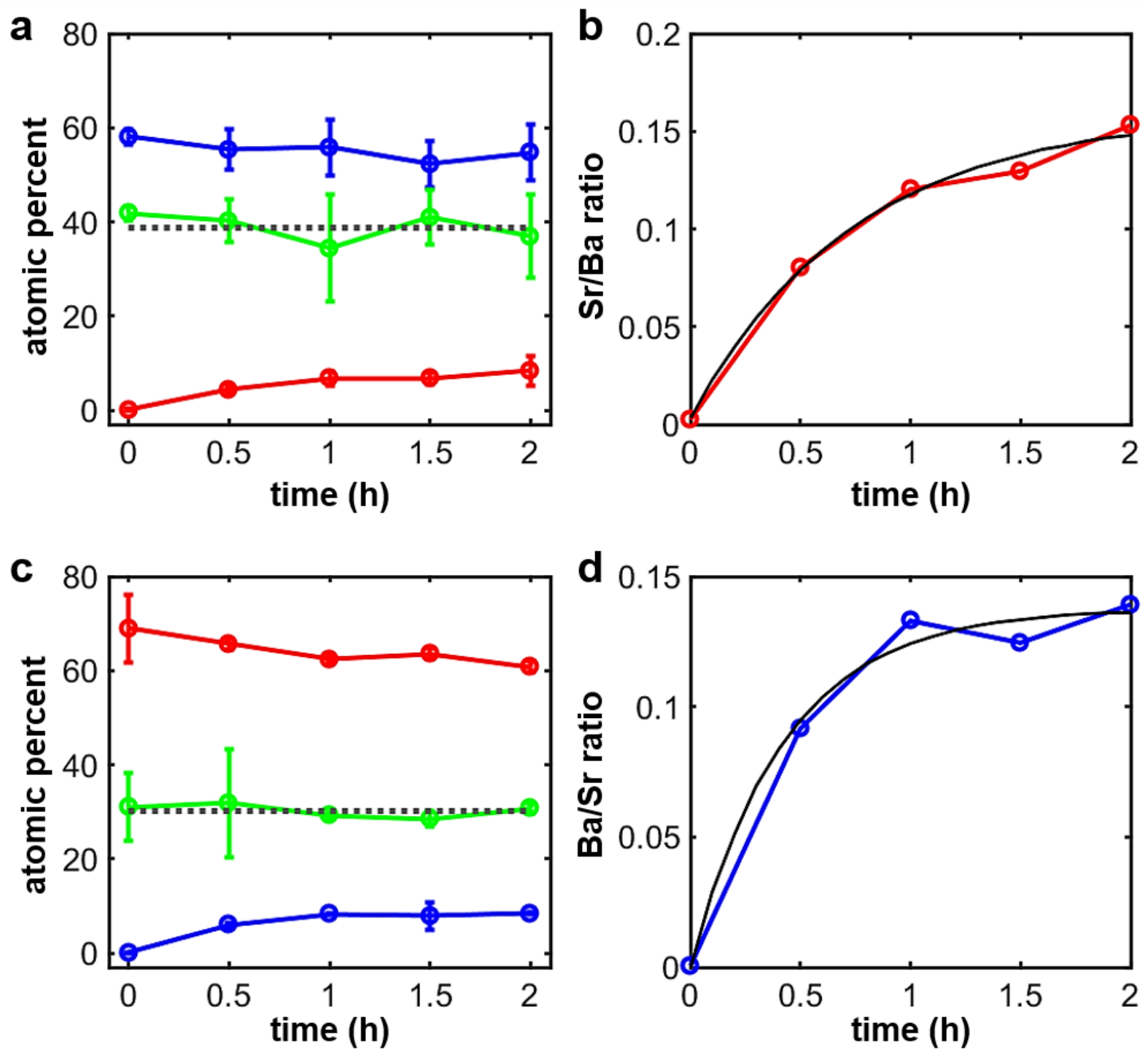

Figure S8. Compositional analysis of inactive biomorph sheets undergoing metal replacement reactions. The data in $\mathrm{a}, \mathrm{b})$ are obtained from biomorphs that were first grown in $\mathrm{a} \mathrm{BaCl}_{2}$-silicate solution and then placed into a comparable $\mathrm{Sr}\left(\mathrm{NO}_{3}\right)_{2}$-silicate solution. The biomorphs analyzed in $\left.\mathrm{c}, \mathrm{d}\right)$ were first grown in a $\mathrm{Sr}\left(\mathrm{NO}_{3}\right)_{2}$-silicate solution and then placed into a comparable $\mathrm{BaCl}_{2}$-silicate solution. The transfer occurred at $t=0$ and the EDX analyses were performed after different waiting times between 0 and $2 \mathrm{~h}$. Blue, green, and red lines and markers in $\mathrm{a}, \mathrm{c}$ ) correspond to $\mathrm{Ba}, \mathrm{Si}$, and $\mathrm{Sr}$, respectively. The dashed lines are the average Si percentage and show that the Si content remained constant during the two hours. The data in $\mathrm{b}, \mathrm{d})$ are the $\mathrm{Sr} / \mathrm{Ba}$ and $\mathrm{Ba} / \mathrm{Sr}$ molar ratios, respectively. The experiments employed plastic substrates to avoid mistakes in the Si characterization that could occur on glass. The continuous black lines are leastsquare fits of the exponential function $A \exp (-k t)+B$. The fit parameters are $A=0.16, B=0.0$, and $k=$ $1.34 \mathrm{~h}^{-1}$ in b) and $A=0.14, B=0.0$, and $k=2.34 \mathrm{~h}^{-1}$ in d). Both saturation values $A$ are smaller than the ratios of secondary-to-primary metal ions ( 0.21 , see Figures $S 7 \mathrm{~b}$ and $4 \mathrm{c}$ and consider the inverse ratios) found near the transition zone (interior side). This difference is interpreted as evidence for secondary crystallization within the gaps between neighboring nanodots. 

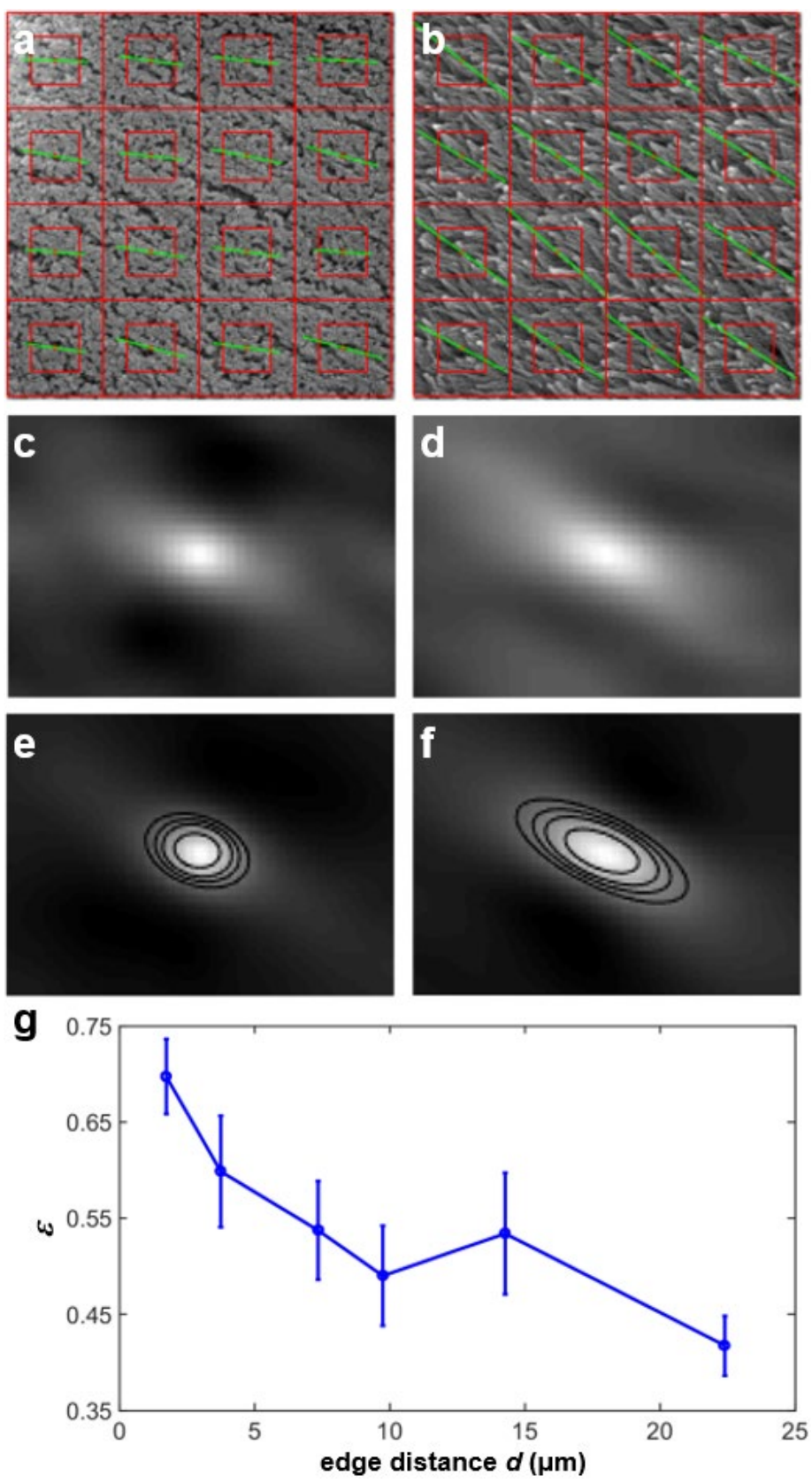

Figure S9. Two-dimensional autocorrelation analysis of SEM images in Figure S1. Examples are given for a) the area closest to the edge showing nanodots and b) an interior area $22.4 \mu \mathrm{m}$ from the edge with prominent nanorod features. The large red boxes in $a, b)$ are the different analyzed areas and the green lines show the main axis of anisotropy. Autocorrelation maps for an individual large red box in a,b) are shown in c,d), respectively. e,f) The mean for all 16 autocorrelation maps with superposed lines of equal correlation. Notice that the curves obtained for nanodots e) are more circular than those for the nanorods f). g) Eccentricity $\varepsilon$ of the latter curves as a function of edge distance. For this analysis, the eccentricity is calculated by fitting ellipses to iso-correlation curves between 0.25 and 0.75 . The corresponding average and standard deviation are graphed as the marker points and error bars, respectively. The eccentricity is defined as the ratio of the ellipses' short and long half axes. The decreasing $\varepsilon$ values indicate an increase in image anisotropy; however, even the nanodot region (image closest to the edge) shows anisotropy. 


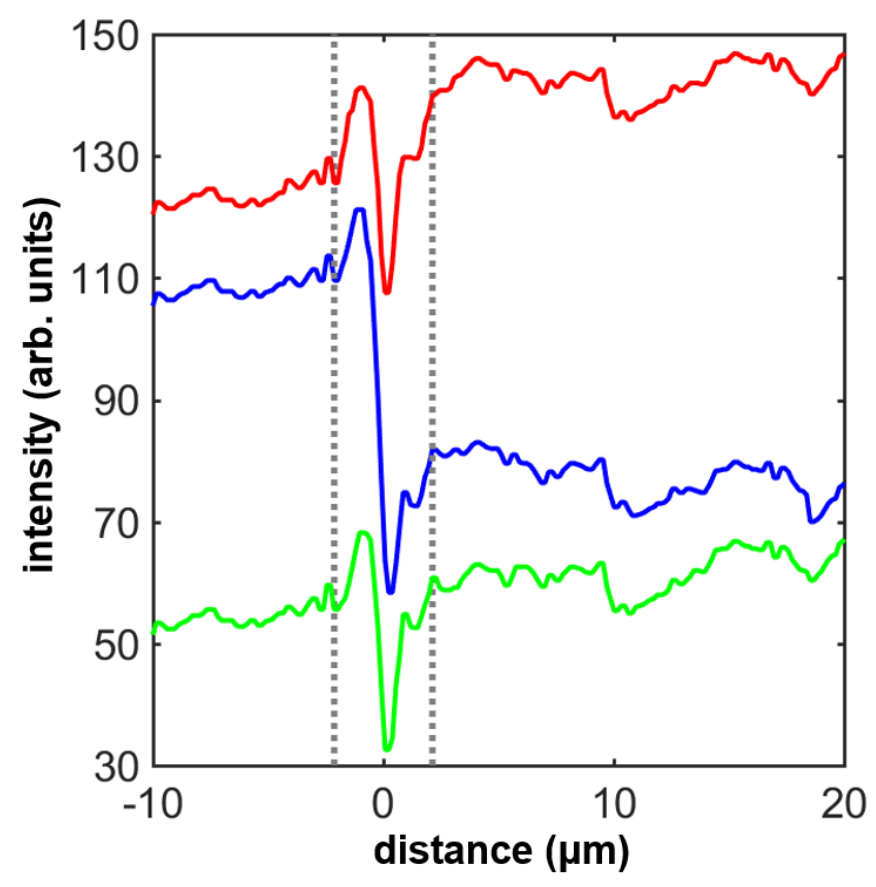

Figure S10. Complete information on the red, green, and blue color channels for the result in Figure 5. In this example, the jump in color intensity is most prominent in the blue channel and occurs over a conservatively estimated distance of $4.3 \mu \mathrm{m}$ (dotted lines).

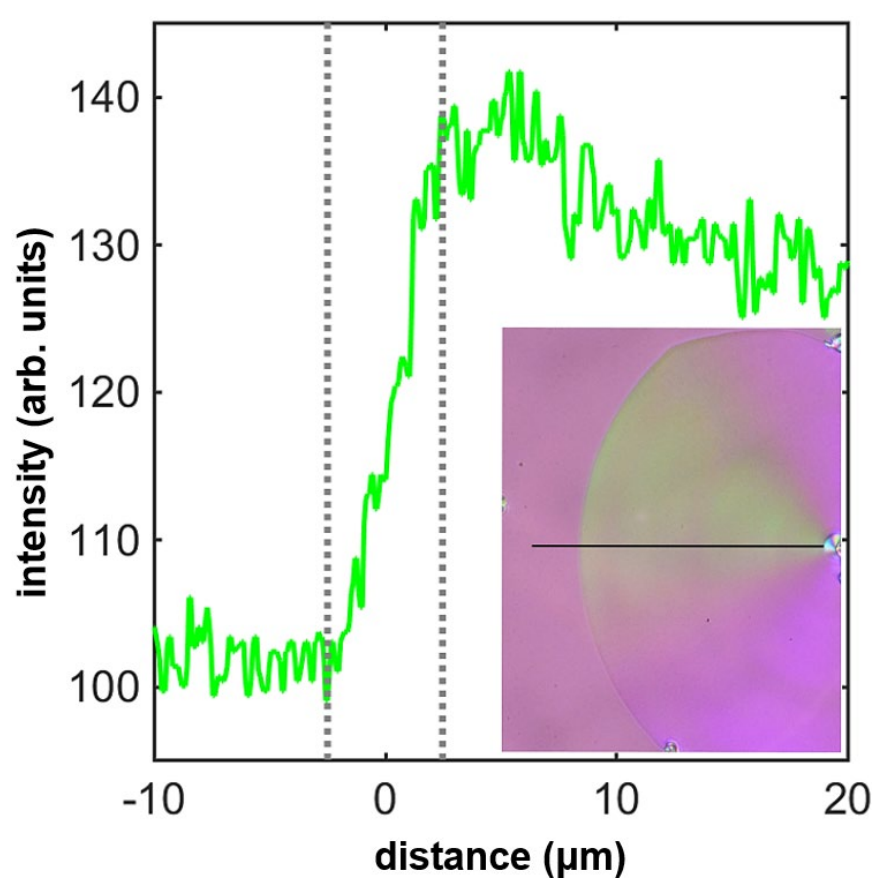

Figure S11. Analysis of a biomorph sheet covered with mineral oil using polarized light microscopy. The inset shows the original micrograph, in which the pink background color arises from strain in the Petri dish. The intensity profile is the green color channel graphed along the black line (inset). The zone for the rise in color intensity measures $5.0 \mu \mathrm{m}$. 


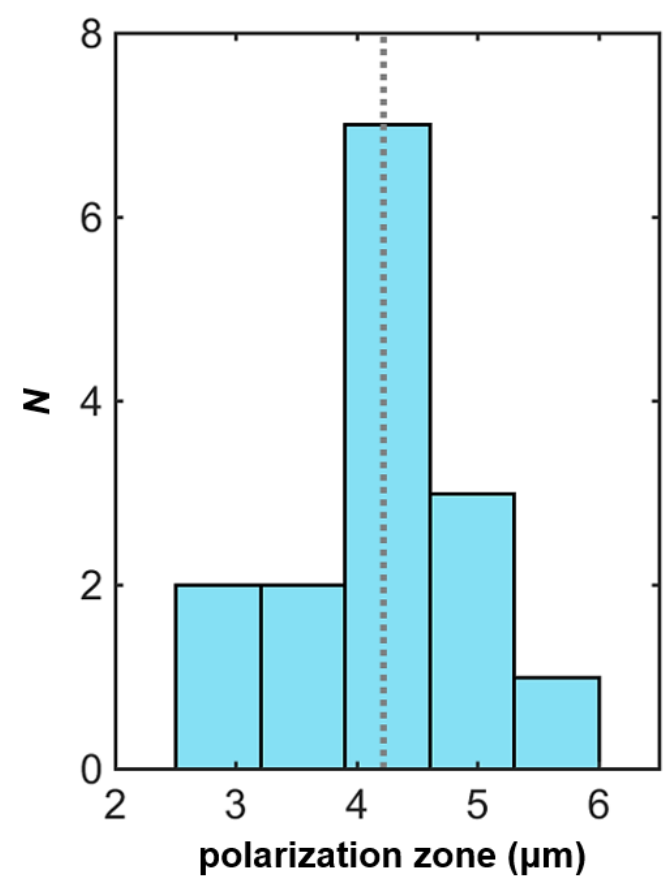

Figure S12. We measured the width of the transition zone for active edges (e.g. Figure S10 and S11) for 15 different biomorph sheets. The results are summarized in this histogram. The mean zone width is (4.2 $\pm 0.8) \mu \mathrm{m}$ and indicated by the dashed gray line.
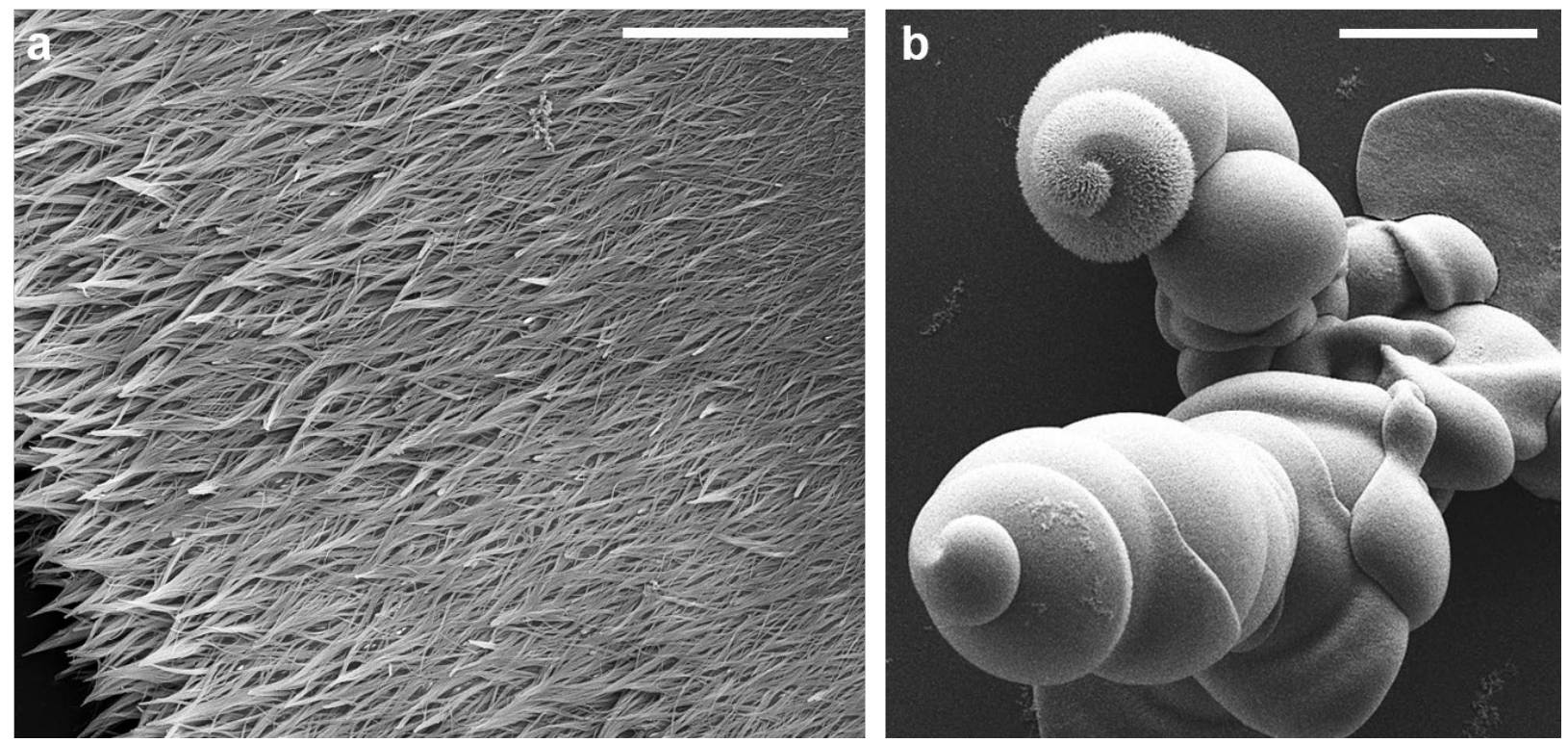

Figure S13. Low magnification SEM images of a) the seaweed-like structures (Figures 6a,b) and b) the biomorph worm (Figure 6c) shown in main text. Notice that the close-up in Figure $6 \mathrm{c}$ is the upper helix in b). Scale bars: a) $3 \mu \mathrm{m}$, b) $20 \mu \mathrm{m}$. 


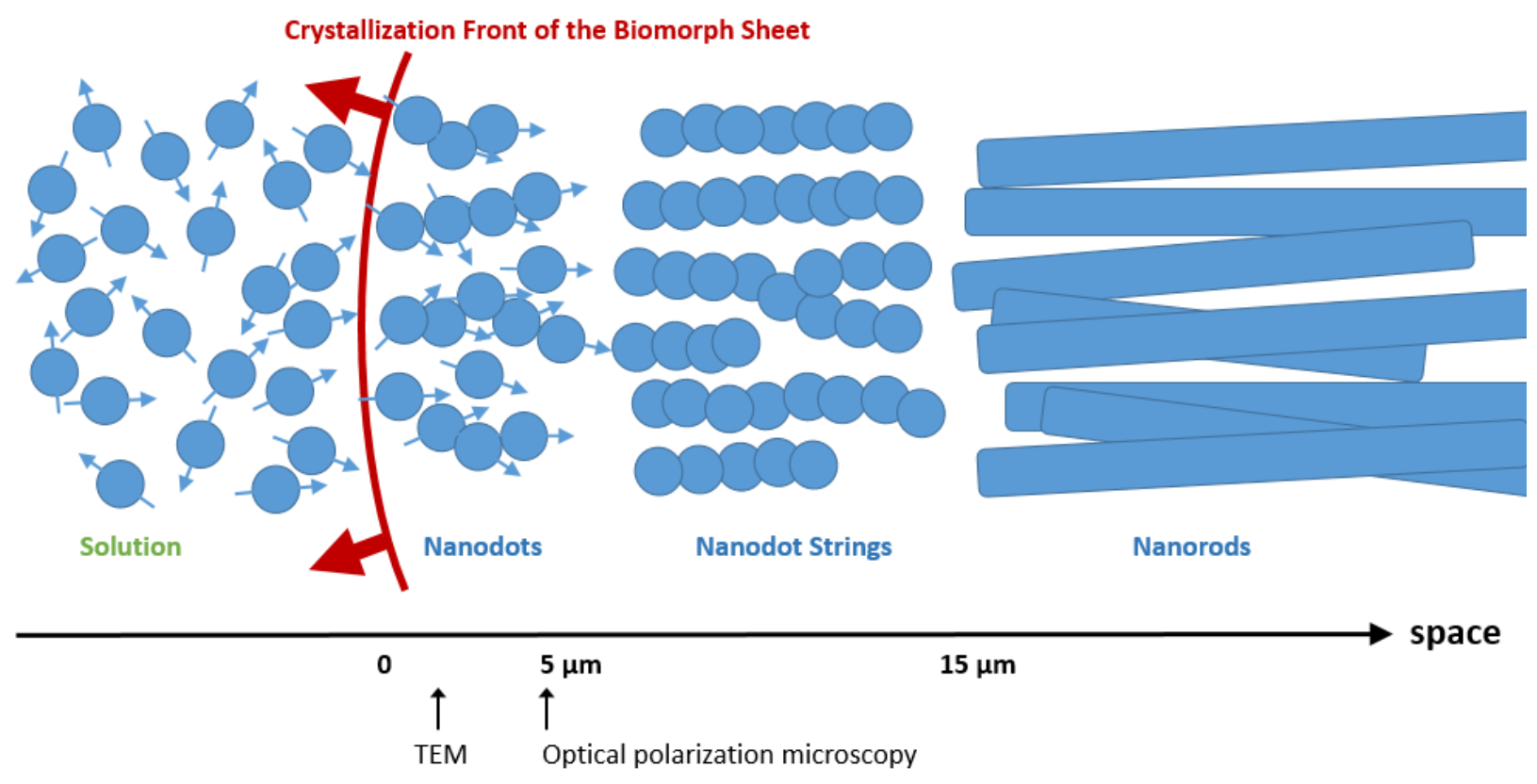

Figure S14. Schematics of the growth and internal transformation processes. The role of possible dipole moments and the degree of alignment during attachment are unclear at this point. 